\title{
Emery-Dreifuss muscular dystrophy type 2 associated (?) with mild peripheral polyneuropathy
}

\author{
Agnieszka Madej-Pilarczyk ${ }^{1}$, Katarzyna Kotruchow ${ }^{1}$, Dagmara Kabzinska ${ }^{1}$, Joanna Cegielska ${ }^{2}$, \\ Andrzej Kochanski ${ }^{1}$, Irena Hausmanowa-Petrusewicz ${ }^{1}$ \\ ${ }^{1}$ Neuromuscular Unit, Mossakowski Medical Research Centre, Polish Academy of Sciences, Warsaw, ${ }^{2}$ Department of Neurology, \\ Medical University of Warsaw, Bielanski Hospital, Warsaw, Poland
}

To our deepest regret Prof. Irena Hausmanowa-Petrusewicz passed away on July 07, 2015.

\begin{abstract}
In recent years numerous mutations in the $L M N A$ gene encoding lamin $A / C$ were shown to segregate with a wide spectrum of phenotypes. A recurrent p.R377H mutation in the LMNA gene was reported in patients with Emery-Dreifuss dystrophy (EDMD2) with various ethnic backgrounds. We present a patient with EDMD2 caused by a p.R377H mutation, associated with mild peripheral polyneuropathy. The analysis of peripheral myelin protein 22 (PMP22), gangliosideinduced differentiation-associated protein 1 (GDAP1), gap junction $\beta$-1 protein (GJB1), and myelin protein zero (MPZ) genes did not reveal mutations; however, we identified a new sequence intronic variant in the mitofusin 2 (MFN2) gene of unknown pathogenic significance. A complex phenotype in the presented patient might depend either on single mutation in the LMNA gene or on bigenic defect; therefore, a wide genetic investigation is needed to elucidate the molecular background of EDMD2/polyneuropathy in this case.
\end{abstract}

Key words: Emery-Dreifuss muscular dystrophy, Charcot-Marie-Tooth neuropathy, LMNA, lamin A/C, mitofusin 2.

\section{Introduction}

Mutations in the LMNA gene have been shown to segregate with a wide spectrum of phenotypes, known as lamiopathies [10], which might manifest as muscular dystrophy, cardiomyopathy, peripheral neuropathy, lipodystrophy, or premature ageing syndromes. In a large cohort of patients with LMNA mutations reported to date, only in few patients a "double" phenotype of axonal neuropathy coexisting with muscular dystrophy $[1,5]$ or dilated cardiomyopathy [4] was reported. In this study we present a patient with genetically confirmed Emery-Drei- fuss dystrophy type 2 (EDMD2), who additionally had late-onset mild, distal, axonal neuropathy. The coexistence of axonal neuropathy and EDMD2 might either result from a single LMNA mutation or have a bigenic background. By analyzing of five genes involved in pathogenesis of polyneuropathy, i.e. peripheral myelin protein 22 (PMP22), ganglioside-induced differentiation-associated protein 1 (GDAP1), gap junction $\beta-1$ protein (GJB1), myelin protein zero (MPZ) and mitofusin 2 (MFN2) genes, we attempted to elucidate the molecular background of axonal polyneuropathy in our EDMD2 patient. 


\section{Case report}

A thirty-four-year-old female (III:6) presented because of slowly progressing walking difficulties and weakness and atrophy of arms and thigh muscles. Since childhood she had been very slim, physically weaker, and less agile than her peers (Fig. 1). During adolescence frequent fainting occurred. Similar clumsy walking was seen in the proband's father (II:4) and her grandmother (I:1); however, they were not examined neurologically. In addition, both relatives had a pacemaker implanted due to conduction disturbances, and the same cardiac problem occurred in the patient's uncle (II:2), who otherwise did not have any skeletal muscle disease. All three of these persons died suddenly at the age of 58 years (II:4), 62 years (II:2), and 66 years (I:1), respectively. Awareness of positive family history for a life-threatening cardiac disease and muscle symptoms being a cause of progressive physical disability were the main reasons for the proband to seek medical attention. Neurological examination revealed atrophy of arms, thighs, and calf muscles, weakness of proximal muscles with Gowers' sign, iliac asymmetry, absence of knee and ankle reflexes, spine rigidity with lumbar hyperlordosis, and slight elbow, knee, and ankle contractures. Superficial sensation was decreased in distal parts of lower limbs ("short socks"), while vibration sensation was normal. 24-hour electrocardiogram (ECG) monitoring revealed numerous episodes of supraventricular tachycardia and periodical atrioventricular block grade I. Echocardiogram was normal. On electroneurography (ENG) the stimulation of the left peroneal nerve revealed trace response in the distal point ( $0.1 \mathrm{mV}$; velocity not recordable) with no response near the fibula head; the stimulation of the right peroneal nerve revealed prolongation of the motor response latency (10.4 ms). Nerve conduction velocities were slightly decreased in the motor fibres of tibial and sural nerves, being $42.5 \mathrm{~m} / \mathrm{s}$ and $45.2 \mathrm{~m} / \mathrm{s}$, respectively. Clinically affected members (I:1, II:2, II:4) were unavailable for ENG/electromyography (EMG), but the examination was normal in the patient's aunt (II:6) and mother (II:3). Once written informed consent and Bioethical Committee approval had been received, we performed genetic analysis in the proband and in her unaffected mother (II:3) and aunt (II:6). Genomic DNA was extracted from peripheral blood lymphocytes using a saltingout procedure. All 12 exons of $L M N A$ and exon-intron

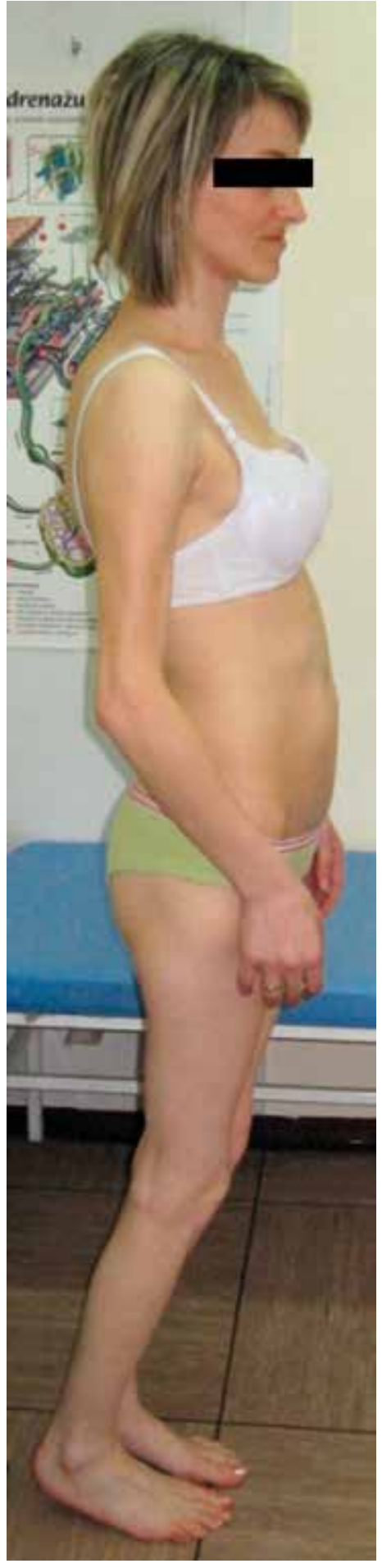

Fig. 1. Thirty four years old patient with EmeryDreifuss dystrophy and mild axonal neuropathy; note atrophy of proximal muscles and slight elbow, knee and ankle contractures. 


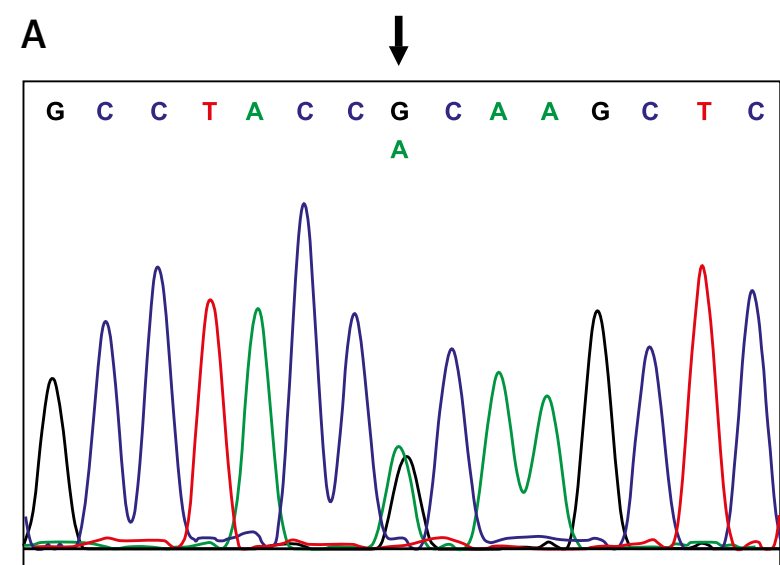

Fig. 2. A) A forward sequencing of $L M N A$ exon 6 revealing heterozygous point mutation $c .1130 \mathrm{G}>\mathrm{A}$ (p.R377H) in proband. B) Pedigree of the proband's family with 3 other affected members, presumptively bearing the same dominant LMNA mutation.
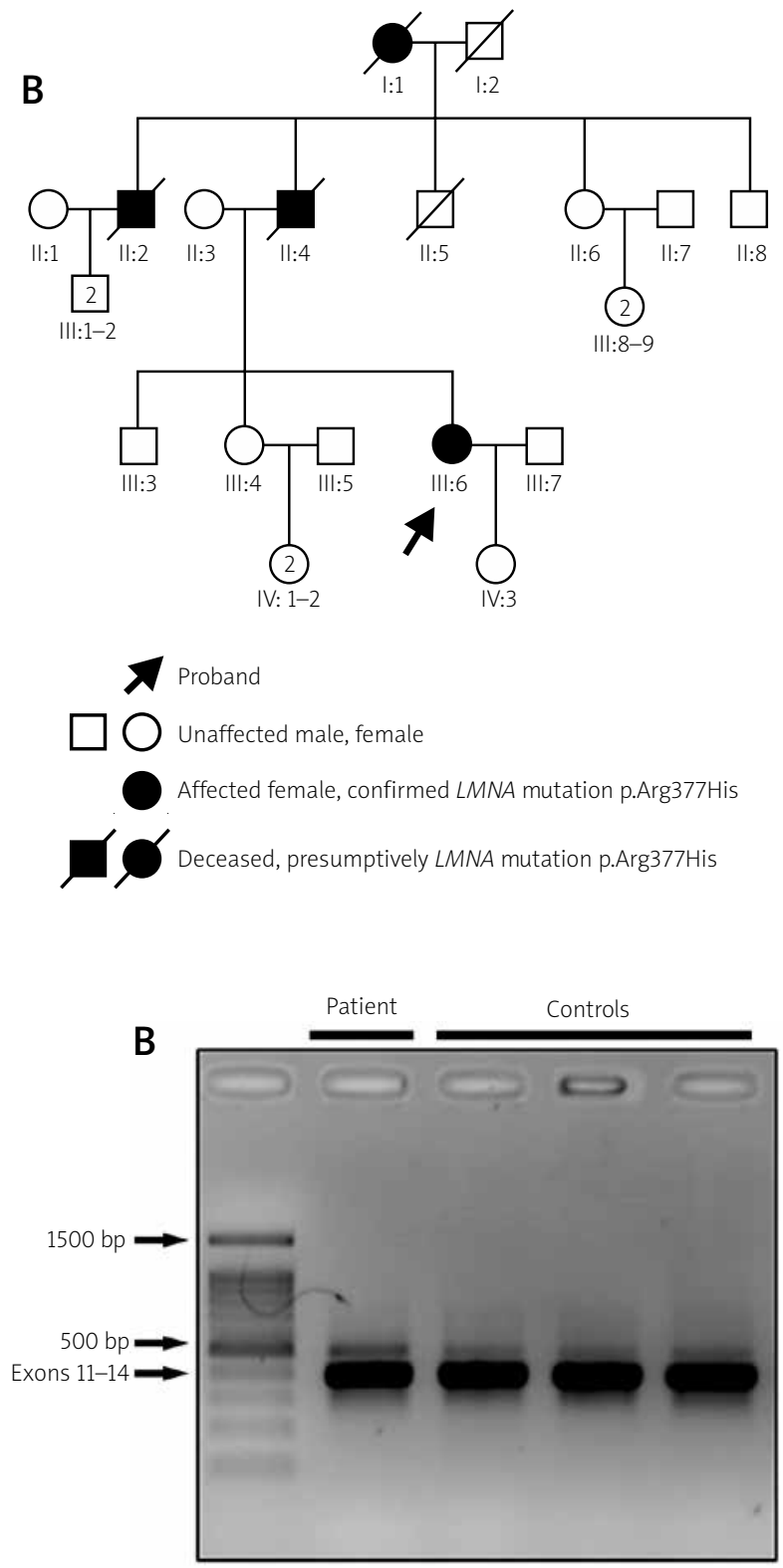

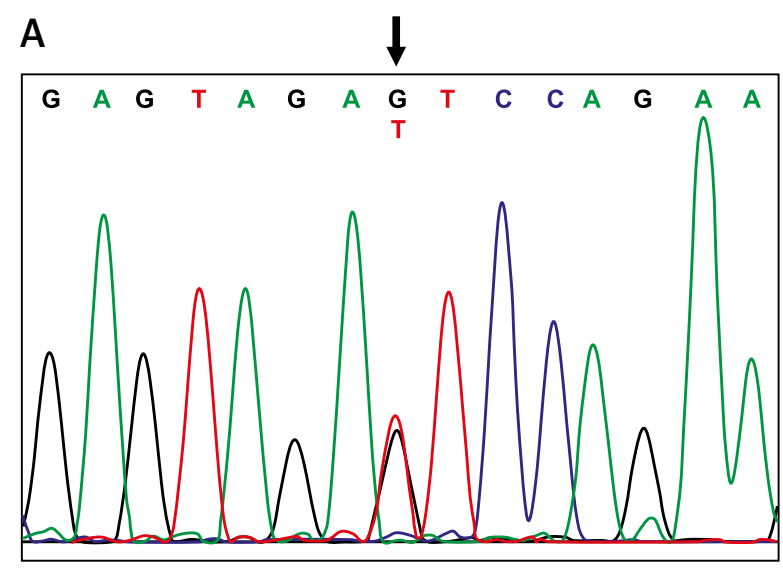

Fig. 3. A) Heterozygous mutation c.1468+50G>T in MFN2 gene. B) PCR amplification of proband's cDNA and control cDNA showing no visible difference in products' length.

junctions were amplified by PCR, sequenced using the Big Dye Terminator Sequencing Ready Reaction kit (Applied Biosystems), and analysed on an ABI PRISM 373 fluorescent DNA sequencer (Applied Biosystems). Analysis of the $L M N A$ gene revealed a heterozygous missense mutation c.1130G >A (p.R377H) (Fig. 2). Analysis of PMP22 did not reveal deletion/ duplication. Direct sequencing of GDAP1, GJB1, and $M P Z$ did not reveal mutations. Sequence analysis of MFN2 showed a single nucleotide substitution
c.1469+50G $>T$ within the twelfth intron, not found either in the proband's unaffected mother (II:3) and aunt (II:6) nor in 100 healthy controls. To check whether it affects splicing, we extracted mRNA from blood lymphocytes and then obtained cDNA from mRNA, encompassing exons 12-13 of MFN2 gene. Polymerase chain reaction (PCR) with the designed primers: forward in 11 exon and reverse in 14 exon, resulted in synthesis of the 391 bp oligonucleotide, which had the same length in the proband and in 
3 healthy controls. This might indicate that MFN2 variant c.1469+50G >T does not affect splicing (Fig. 3).

\section{Discussion}

The coexistence of axonal polyneuropathy with muscular dystrophy/dilated cardiomyopathy has been shown only in a few patients $[1,4,5]$. Laminopathy with peripheral nerve involvement, associated with homozygous LMNA mutation p.R298C, was first described by De Sandre-Giovannoli et al. in 2002 [3]. There have been several reports of laminopathies with overlapping phenotype of axonal neuropathy and muscular dystrophy and/or cardiac involvement with autosomal dominant trait of inheritance. In 2004 Goizet et al. described a 55-year-old man and his 13-year-old daughter presenting axonal neuropathy, muscular dystrophy, cardiac involvement, and leukonychia, in whom LMNA mutation p.E33D was found [5]. Both patients had sensory abnormalities in distal lower limbs, while pes cavus, pelvic and distal muscle weakness was seen only in the older one. In 2005 Benedetti et al. reported a boy with LMNA mutation c.1711C $>\mathrm{T}$, who had progressive distal and proximal muscle atrophy/weakness, causing him to be wheelchair bound; as well as pes equinovarus, which was corrected surgically, mildly impaired proprioceptive sensation and fat accumulation in the face and neck were seen [1]. A cardio-neurological form of laminopathy, associated with LMNA mutation c.1496delC, has been described in a 40-year-old man by Duparc et al. [4]. In addition to heart disease atrophy of quadriceps muscle, lack of reflexes, and contracted feet were found and ENG showed moderate axonal neuropathy. However, no molecular basis of EDMD2/Charcot-Marie-Tooth neuropathy (CMT) coexistence has been identified. The question remains unanswered: whether the phenotype in the presented case results from a single mutation within the LMNA gene or rather represents an overlapping syndrome associated with mutations in at least two genes. Our patient harbours a mutation in LMNA, c.1130G>A (p.R377H). This has been shown to segregate with phenotypes affecting skeletal and cardiac muscles, i.e. EDMD2 [8], limb-girdle muscular dystrophy 1B (LGMD1B) [6], isolated cardiomyopathy with conduction defects (DCM-CD) [7], or quadriceps myopathy with DCM-CD [2]. No axonal neuropathy in EDMD2 was reported as being caused by $\mathrm{R} 377 \mathrm{H}$ mutation in the LMNA gene. To elucidate the genetic background of the disease in our patient we analysed five genes: PMP22, GDAP1, GJB1, MPZ, and MFN2, causative for CMT disease. Heterozygous mutations in GDAP1 segregate with a mild form of axonal neuropathy with slow progression, resembling the clinical course observed in our patient [11]. Analysis of the GDAP1 gene in our patient did not reveal mutations. Also, no mutations in the coding sequence of PMP22, GJB1, and MPZ genes were found. The MFN2 gene is the most commonly mutated gene in autosomal dominant CMT2. Late onset, milder phenotype is associated either with intronic mutations or with mutations at the end of the coding sequence of the MFN2 gene [9]. We attempted to investigate the pathogenic status of the sequence variant C.1469+50G >T in MFN2; the analysis of cDNA showed that it probably did not affect splicing (Fig. 2). We cannot definitively exclude that another unknown mutation caused the peripheral neuropathy observed in our patient. Polyneuropathy has never been reported in the context of the LMNA mutation p.R377H; therefore, bigenic molecular pathogenesis seems more likely in the presented case of EDMD2/CMT2. Initially we decided to sequence the late onset-CMT2-associated genes instead of exome sequencing analysis because hundreds of NGS-generated variants of unknown clinical relevance might be difficult to interpret. Since no mutations were detected in the analysed genes responsible for neuropathy, it seems that only wide genetic analysis could help to clarify the genetic basis of this case.

\section{Acknowledgements}

This study was supported by a grant from the Polish National Science Centre No. 2012/07/B/NZ4/ 01748 to DK. The authors would like to thank Ms Jadwiga Kędzierska for her technical assistance.

\section{Disclosure}

Authors report no conflict of interest.

\section{References}

1. Benedetti S, Bertini E, lannaccone S, Angelini C, Trisciani M, Toniolo D, Sferrazza B, Carrera P, Comi G, Ferrari M, Quattrini A, Previtali SC. Dominant LMNA mutations can cause combined muscular dystrophy and peripheral neuropathy. J Neurol Neurosurg Psychiatry 2005; 76: 1019-1021.

2. Charniot JC, Desnos M, Zerhouni K, Bonnefont-Rousselot D, Albertini JP, Salama JZ, Bassez G, Komajda M, Artigou JY. Severe 
dilated cardiomyopathy and quadriceps myopathy due to lamin A/C gene mutation: a phenotypic study. Eur J Heart Fail 2006; 8: 249-256.

3. De Sandre-Giovannoli A, Chaouch M, Kozlov S, Vallat JM, Tazir M, Kassouri N, Szepetowski P, Hammadouche T, Vandenberghe A, Stewart CL, Grid D, Lévy N. Homozygous defects in LMNA encoding lamin A/C nuclear-envelope proteins, cause autosomal recessive axonal neuropathy in human. Am J Hum Genet 2002; 70: 726-736.

4. Duparc A, Cintas P, Somody E, Bieth E, Richard P, Maury P, Delay M. A cardio-neurological form of laminopathy: dilated cardiomyopathy with permanent partial atrial standstill and axonal neuropathy. Pacing Clin Electrophysiol 2009; 32: 410-415.

5. Goizet C, Yaou RB, Demay L, Richard P, Bouillot S, Rouanet M, Hermosilla E, Le Masson G, Lagueny A, Bonne G, Ferrer X. A new mutation of the lamin $A / C$ gene leading to autosomal dominant axonal neuropathy, muscular dystrophy, cardiac disease, and leuconychia. Med Genet 2004; 41: e29.

6. Muchir A, Bonne G, van der Kooi AJ, van Meegen M, Baas F, Bolhuis PA, de Visser M, Schwartz K. Identification of mutations in the gene encoding lamins $\mathrm{A} / \mathrm{C}$ in autosomal dominant limb girdle muscular dystrophy with atrioventricular conduction disturbances (LGMD1B). Hum Mol Genet 2000; 9: 1453-1459.

7. Perrot A, Sigusch HH, Nägele H, Genschel J, Lehmkuhl H, Hetzer R, Geier C, Leon Perez V, Reinhard D, Dietz R, Josef Osterziel K, Schmidt HH. Genetic and phenotypic analysis of dilated cardiomyopathy with conduction system disease: demand for strategies in the management of presymptomatic lamin A/C mutant carriers. Eur J Heart Fail 2006; 8: 484-493.

8. van Tintelen JP, Hofstra RM, Katerberg H, Rossenbacker $T$ Wiesfeld AC, du Marchie Sarvaas GJ, Wilde AA, van Langen IM Nannenberg EA, van der Kooi AJ, Kraak M, van Gelder IC, van Veldhuisen DJ, Vos Y, van den Berg MP; Working Group on Inherited Cardiac Disorders, line 27/50, Interuniversity Cardiology Institute of The Netherlands. High yield of LMNA mutations in patients with dilated cardiomyopathy and/or conduction disease referred to cardiogenetics outpatient clinics. Am Heart J 2007; 154: 1130-1139.

9. Verhoeven K, Claeys KG, Züchner S, Schröder JM, Weis J, Ceuterick C, Jordanova A, Nelis E, De Vriendt E, Van Hul M, Seeman P, Mazanec R, Saifi GM, Szigeti K, Mancias P, Butler IJ, Kochanski A, Ryniewicz B, De Bleecker J, Van den Bergh P, Verellen C, Van Coster R, Goemans N, Auer-Grumbach M, Robberecht W, Milic Rasic V, Nevo Y, Tournev I, Guergueltcheva V, Roelens F, Vieregge P, Vinci P, Moreno MT, Christen HJ, Shy ME, Lupski JR, Vance JM, De Jonghe P, Timmerman V. MFN2 mutation distribution and genotype/phenotype correlation in Charcot-Marie-Tooth type 2 Brain 2006; 129: 2093-2102.

10. Worman HJ, Bonne G. "Laminopathies": a wide spectrum of human diseases. Exp Cell Res 2007; 313: 2121-2123.

11. Zimon M, Baets J, Fabrizi GM, Jaakkola E, Kabzińska D, Pilch J, Schindler AB, Cornblath DR, Fischbeck KH, Auer-Grumbach M, Guelly C, Huber N, De Vriendt E, Timmerman V, Suter U, Hausmanowa-Petrusewicz I, Niemann A, Kochański A, De Jonghe P, Jordanova A. Dominant GDAP1 mutations cause predominantly mild CMT phenotypes. Neurology 2011; 77: 540-548. 